\title{
Comparison of the protein expression of calpain-1, calpain-2, calpastatin and calmodulin between gastric cancer and normal gastric mucosa
}

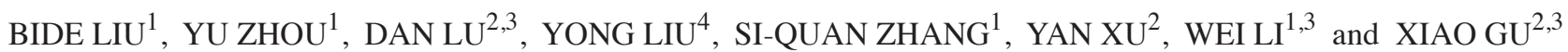 \\ ${ }^{1}$ College of Medicine, ${ }^{2}$ College of Clinical Medicine, Yangzhou University; ${ }^{3}$ Jiangsu Key Laboratory of Integrated \\ Traditional Chinese and Western Medicine for Prevention and Treatment of Senile Diseases, Yangzhou, Jiangsu 225001; \\ ${ }^{4}$ School of Life Science and Medicine, Dalian University of Technology, Panjin, Liaoning 124221, P.R. China
}

Received October 5, 2015; Accepted April 13, 2017

DOI: $10.3892 / \mathrm{ol} .2017 .6617$

\begin{abstract}
The understanding of molecular mechanisms that are involved in the development and the progression of gastric cancer (GC) are of importance for the diagnosis and treatment. The calpain system, which contains the calpains and the endogenous inhibitor, has been suggested as an important factor in the tumorigenesis and migration of colorectal adenocarcinoma, breast and ovarian cancer, and as a prognostic marker for GC. However, the expression level of calpain system proteins in GC and normal-appearing peritumoral gastric mucosa remain unknown. The present study investigated the expression of calpain-1 (CAPN1), calpain-2 (CAPN2), calpastatin and calmodulin (CaM) in GC and uninvolved gastric mucosa tissues with immunohistochemistry. Results demonstrated that CAPN2 protein level increased in GCs compared with normal tissues, while calpastatin and CaM protein level decreased. No evident alterations were observed for CAPN1. Although the protein expression of all these four proteins was not in association with the clinical variables of GC in the present study, higher calpain enzyme activity could be a negative prognostic marker, since calpains are responsible for the generation of active forms of certain proteins that facilitate the progression of cancer. The ratio of (CAPN1 x CAPN2)/(calpastatin x CaM) may serve as a potential index for diagnosis of GC.
\end{abstract}

\section{Introduction}

Gastric cancer (GC) remains a major public health problem, since it was the fourth most common type of cancer and the

Correspondence to: Dr Wei Li, College of Medicine, Yangzhou University, 11 Huaihai Road, Yangzhou, Jiangsu 225001, P.R. China E-mail: weili@yzu.edu.cn

Dr Xiao Gu, College of Clinical Medicine, Yangzhou University, 11 Huaihai Road, Yangzhou, Jiangsu 225001, P.R. China

E-mail: guxiao222@hotmail.com

Key words: gastric cancer, calpain-1, calpain-2, calpastatin, calmodulin third most common cause of cancer-associated mortality worldwide in 2012 (1). In China, GC is the third frequently diagnosed cancer and the third leading cause of cancer-associated mortality in 2011 (2). The understanding of molecular mechanisms involved in the development and the progression of GC is important for the diagnosis and treatment of this disease.

Calpains are a group of calcium-activated, non-lysosomal neutral cysteine proteases (3). At present, the best-characterized calpain isoforms are calpain-1 ( $\mu$-calpain or CAPN1) and calpain-2 (m-calpain or CAPN2), which require $\mu \mathrm{M}$ and $\mathrm{mM}$ concentrations of $\mathrm{Ca}^{2+}$ for their intracellular activity, respectively (3). CANP1 and CAPN2 are composed of a distinct large catalytic subunit $(80 \mathrm{kDa})$ and a small common regulatory subunit (28 kDa) (3). Calpains are responsible for the cleavage of a broad spectrum of protein substrates, resulting in the generation of functional fragments rather than total degradation products $(4,5)$. It has been confirmed that calpains are involved in cell differentiation, migration and transformation, and perform an important role in cancer pathogenesis and progression $(4,6,7)$.

The proteolytic activity of calpain is inhibited by an endogenous inhibitor, calpastatin, in a substrate-competitive manner (8). The expression level of calpastatin directly effects the calpain activity (9). In addition, calmodulin (CaM) is another endogenous regulator of proteolytic activity of calpains (10). By binding to the PEST sequence [a sequence that is rich in proline $(\mathrm{P})$, glutamic acid $(\mathrm{E})$, serine $(\mathrm{S})$ and threonine $(\mathrm{T})$ ], which is the target sequence of calpain, $\mathrm{CaM}$ protects the protein to be cleaved by calpain (11-14).

The role that calpain system performs in cancer is complicated. Calpain has been reported to be involved in apoptosis of cancer cells (15). In addition, the upregulation of calpain may be involved in cancer development and migration (16), and calpain expression is upregulated in a number of tumors, including colorectal adenocarcinoma (17), breast cancer (18) and ovarian cancer (19). In GC, it has been reported that the calpain activity was upregulated compared with normal tissue (20), and it is speculated that higher calpain and calpastatin expression may be a favorable prognostic marker for GC (21). However, there is still no direct experimental 
data about the comparison of the protein expression levels of the members of calpain system in GC and normal gastric mucosa.

In order to understand the roles that calpain, calpastatin and CaM serve in GC, the current study compared the expression levels of CAPN1, CAPN2, calpastatin and CaM in human GC tissues and normal glandular tissues with immunohistochemistry. The present results demonstrated that CAPN2 protein level increased in GC compared with normal tissue, while calpastatin and CaM protein level decreased. Calpain-1 appeared unchanged. None of the protein expression levels were found to be associated with the clinical variables of GC in the present study. The ratio of (CAPN1 $x$ CAPN2)/(calpastatin x CaM) best predicted GC.

\section{Materials and methods}

Materials. Polyclonal antibodies specific for CANP1 (dilution, 1:1,000; cat no. PA5-27681), CAPN2 (dilution, 1:1,000; PA5-27720) and CaM (cat no. MA3-917; 1:20) were obtained from Thermo Fisher Scientific, Inc. (Waltham, MA, USA) and anti-calpastatin (cat no. sc-20779; 1:500) was purchased from Santa Cruz Biotechnology, Inc. (Dallas, TX, USA). Immunol Staining Primary Antibody Diluent Buffer and Hematoxylin were from Nanjing KeyGen Biotech Co., Ltd. (Nanjing, China). UltraSensitive ${ }^{\mathrm{TM}} \mathrm{S}-\mathrm{P}$ kit and 3,3'-diaminobenzidine (DAB) kit were purchased from Fuzhou Maixin Biotech Co., Ltd. (Fuzhou, China).

Clinical samples. Cancerous tissues and normal peritumoral gastric mucosa (5 $\mathrm{cm}$ away from the tumor) were collected from patients $(\mathrm{n}=51)$ treated at Northern Jiangsu People's Hospital (Yangzhou, China) between January 2014 and December 2015. The age and gender distribution, and the clinicopathological variables of the patients are summarized in Table I. The study design was approved by the local ethics committee of Northern Jiangsu People's Hospital, in accordance with the guidelines of the 1975 Declaration of Helsinki. Written informed consent was obtained from all patients. Surgical pathology specimens of 51 patients with GC who had undergone resection were collected directly from surgery and fixed in formalin (10\%) immediately at $4^{\circ} \mathrm{C}$ for $24 \mathrm{~h}$. Normal-appearing peritumoral gastric mucosa was similarly dissected and fixed.

Immunohistochemistry. Sections $(4 \mu \mathrm{m})$ of the formalin-fixed, paraffin-embedded tissues were cut, followed by deparaffinizing in xylene and rehydrating in an alcohol gradient. Antigen retrieval was performed in citrate buffer $(\mathrm{pH} 6.0)$ using an electric pressure cooker for $2 \mathrm{~min}$ at $120^{\circ} \mathrm{C}$, and cooled naturally in the buffer for $20 \mathrm{~min}$. Immunostaining was performed according to the streptavidin-peroxidase method using a kit (Nanjing KeyGen Biotech Co., Ltd.) according to the manufacturer's protocol. Subsequent to blocking of the samples with blocking solution in the kit, the primary antibodies anti-CAPN1 (dilution, 1:1,000; cat no. PA5-27681; Thermo Fisher Scientific, Inc.), anti-CAPN2 (dilution, 1:1,000; cat no. PA5-27720; Thermo Fisher Scientific, Inc.), anti-calpastatin (dilution, 1:500; cat no. sc-20779; Santa Cruz Biotechnology, Inc.) and anti-CaM (1:20; cat no. MA3-917; Thermo Fisher Scientific, Inc.) were diluted using primary
Table I. Clinicopathological variables of patient sample.

\begin{tabular}{lc}
\hline Variables & Patients, $\mathrm{n}$ \\
\hline Age, years (mean \pm SD) & $62.5 \pm 10.1$ \\
Gender & \\
Male & 41 \\
Female & 10 \\
Tumor size, $\mathrm{cm}^{3}$ (mean \pm SD) & $6.4 \pm 1.6$ \\
T classification & \\
1 & 1 \\
2 & 6 \\
3 & 39 \\
4 & 5 \\
N classification & \\
0 & 10 \\
1 & 16 \\
2 & 20 \\
3 & 5 \\
Clinical stage & \\
I & \\
II & \\
IIIa & 16 \\
IIIb & 7 \\
IV & 7 \\
\hline
\end{tabular}

$(n=51) . S D$, standard deviation; T, tumor; N, node.

antibody diluents and applied to the tissues at $4^{\circ} \mathrm{C}$ overnight. Staining was achieved using the DAB dye for $1 \mathrm{~min}$ at $25^{\circ} \mathrm{C}$.

The staining was observed with an upright microscope (magnification, x40, 10 fields of view/slice) equipped with a CCD camera (Olympus Corporation, Tokyo, Japan). The images were document with cellSens Entry software (Ver. 1.5; Olympus Corporation, Tokyo, Japan). The expression of the four proteins was quantified by mean optical density (MOD) using Image-Pro Plus 6.0 software (Media Cybernetics, Inc., Rockville, MD, USA).

Statistical analysis. The results were presented as mean \pm standard deviation. Based on whether the distribution was normal or not, paired t-test or Wilcoxon matched-pairs signed rank test were used to compare the MOD variance in cancer and its peritumoral normal mucosa tissues. The association between calpain system protein expressions with each other was subjected to Pearson's rank correlation. Receiver operator characteristic (ROC) curves were used to investigate which protein could be the best indicator for $\mathrm{GC}$, and the cut-off points were generated according to ROC curve and Youden's index. The association between protein expression and clinicopathological variables was assessed using unpaired t-test (gender) and Spearman's rank correlation (other parameters). $\mathrm{P}<0.05$ was considered to indicate a statistically significant difference. Statistical analysis was performed using SPSS 17.0 software (SPSS, Inc., Chicago, IL, USA). 


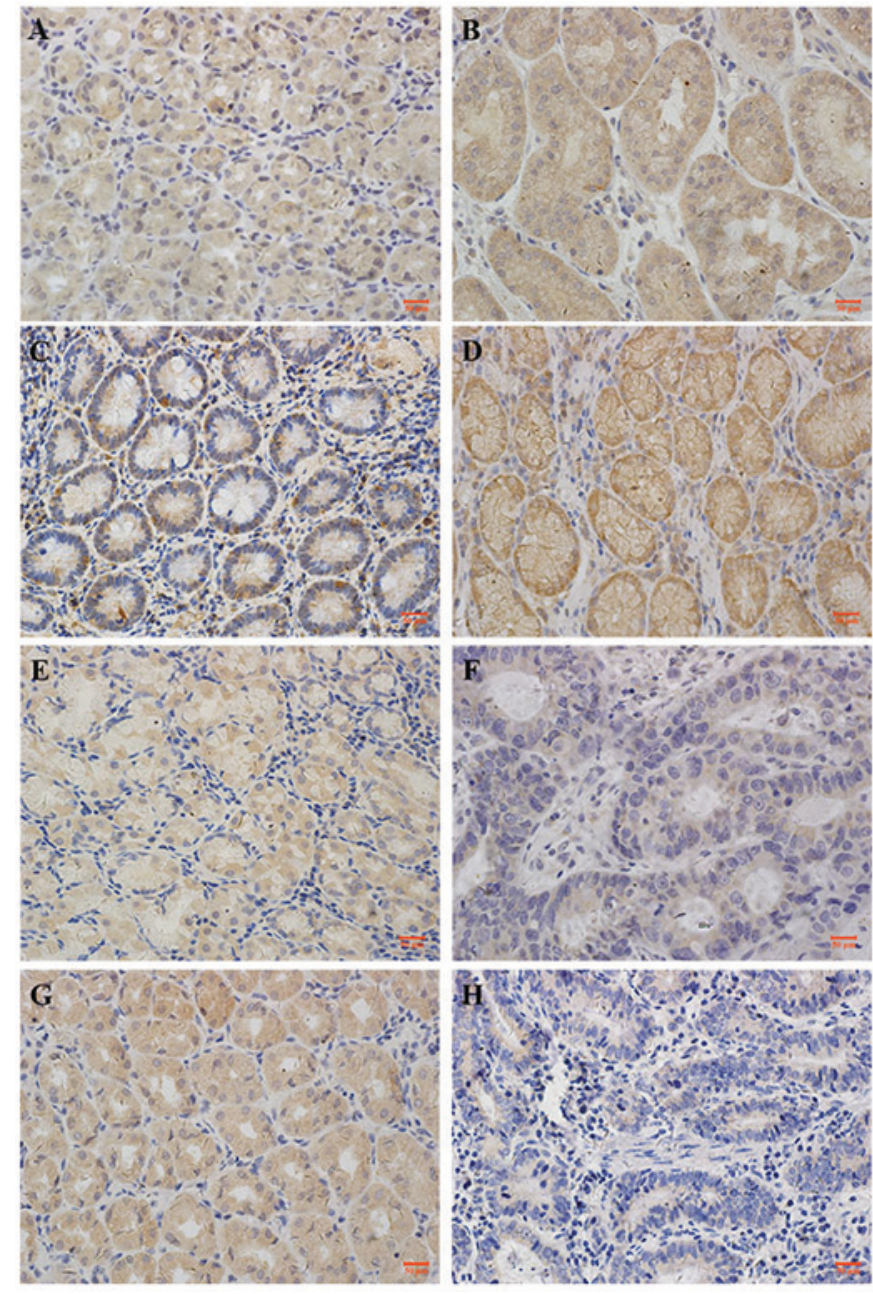

Figure 1. Representative photomicrographs of proteins expression levels. (A) CAPN1 expression in normal tissue. (B) CAPN1 expression in GC tissue. (C) CAPN2 expression in normal tissue. (D) CAPN2 expression in GC tissue. (E) Calpastatin expression in normal tissue. (F) Calpastatin expression in $\mathrm{GC}$ tissue. (G) CaM expression in normal tissue. (H) CaM expression in $\mathrm{GC}$ tissue. Photomicrographs are at $\mathrm{x} 40$ magnification, where the scale bar shows $50 \mu \mathrm{m}$. GC, gastric cancer; CaM, calmodulin.

\section{Results}

Stain location and frequency. CAPN1, CAPN2, calpastatin and $\mathrm{CaM}$ demonstrated cytoplasmic staining, with a certain degree of heterogeneity within samples. Typical staining patterns are shown in Fig. 1. In normal tissues, CAPN1 had a median MOD value of $2.7 \times 10^{-2}$, and ranged between $2.9 \times 10^{-3}$ to $6.1 \times 10^{-2}$; CAPN2 had a median MOD value of $5.0 \times 10^{-2}$ and ranged between $1.8 \times 10^{-2}$ to $1.1 \times 10^{-1}$; calpastatin had a median MOD value of $3.3 \times 10^{-2}$ and ranged between $1.9 \times 10^{-3}$ to 9.1 $\times 10^{-2}$; and CaM had a median MOD of $1.2 \times 10^{-2}$ and ranged between $2.0 \times 10^{-8}$ to $8.1 \times 10^{-2}$. In GC tissues, CAPN1 had a median MOD value of $3.2 \times 10^{-2}$ and ranged between $3.2 \times 10^{-3}$ to $1.4 \times 10^{-1}$; CAPN2 had a median MOD value of $8.1 \times 10^{-2}$ and ranged between $2.0 \times 10^{-2}$ to $1.4 \times 10^{-1}$; calpastatin had a median MOD value of $1.7 \times 10^{-2}$ and ranged between $2.8 \times 10^{-3}$ to 6.6x10-2; and CaM had a median MOD of $1.6 \times 10^{-3}$ and ranged between $1.0 \times 10^{-8}$ to $4.3 \times 10^{-2}$. Paired t-test demonstrated that calpain-2 exhibited higher scores in GC compared with the normal tissue (Fig. 2B), while the expression of calpastatin and
A

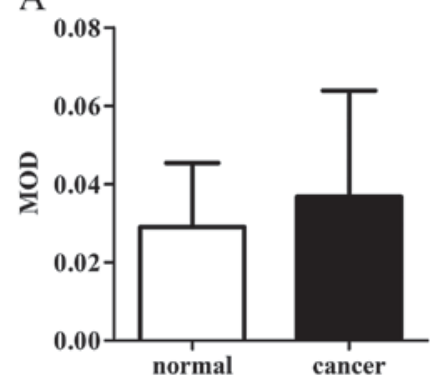

$\mathrm{C}$

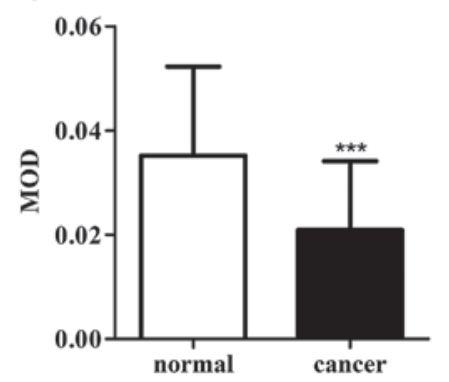

B

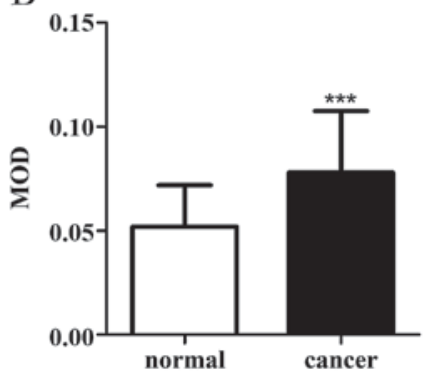

D

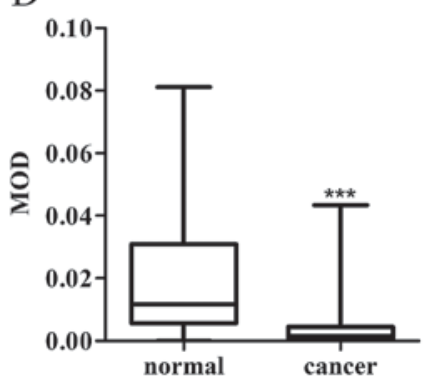

Figure 2. (A) CAPN1, (B) CAPN2, (C) calpastatin and (D) CaM protein expression levels (exhibited as MOD) in gastric cancer and normal tissues $(\mathrm{n}=51) .{ }^{* * *} \mathrm{P}<0.0001$. MOD, mean optical density; CaM, calmodulin.

CaM decreased in cancer tissues (Fig. 2C and D). Wilcoxon matched-pairs signed rank test indicated that the expression of calpain-1 exhibited no difference in GC tissues against gastric mucosa (Fig. 2A). The association between the expression of the proteins with each other was assessed using the Pearson's rank correlation coefficient. As exhibited in Table II, in GC tissues, the expression of CAPN1, CAPN2, calpastatin and $\mathrm{CaM}$ proteins significantly correlated with each other $(\mathrm{P}<0.05)$, though these correlations were weak. In normal-appearing peritumoral gastric mucosa, the expression of these four proteins also correlated with each other. The expression of calpastatin exhibited a stronger correlated with calpain-1 $(r=0.741 ; \mathrm{P}<0.001)$, while that of the others were weak.

\section{Decisions}

ROC curves. ROC curves were employed to determine whether the calpain system proteins are potential GC specific biomarkers. Area under the curve (AUC) of ROC curves, cut-off points and positive likelihood ratio were generated (Table III). Among the four proteins, $\mathrm{CaM}$ appeared to be the best discriminating marker for $\mathrm{GC}(\mathrm{AUC}=83.2 \%$ ). Since the calpain activity is regulated by calpastatin and the activity modulator $\mathrm{CaM}$, the ratios of calpains to the regulators were also investigated (Table III). The results demonstrated that when the ratios of these protein levels were used, the AUCs of ROC curves increased. The MOD value ratio of (CAPN1 xCAPN2)/(calpastatin $\times \mathrm{CaM}$ ) demonstrated the best discrimination power $(\mathrm{AUC}=89.7 \%)$ compared with the situation when single protein expression was used (Table III). Since the ratio of (CAPN1 x CAPN2)/(calpastatin x CaM) may better reflect the calpain activity, calpain activity has the potential to be developed as a new diagnostic indicator for GC.

Association with clinicopathological criteria. The expression levels of CAPN1,CAPN2, calpastatin and CaM were assessed for 
Table II. The correlation between expression of the proteins in normal-appearing peritumoral gastric mucosa and cancer tissue with each other.

\begin{tabular}{lcccc}
\hline & \multicolumn{2}{c}{ Normal tissue } & & Cancer tissue \\
\cline { 2 - 4 } Protein & Pearson value & P-value & Pearson value \\
\hline Calpain-1 with calpaspatin & 0.741 & $<0.0001$ & 0.342 \\
Calpain-2 with calpaspatin & 0.535 & $<0.0001$ & 0.553 \\
CaM with calpaspatin & 0.551 & $<0.0001$ & 0.396 \\
Calpain-1 with calpain-2 & 0.419 & 0.002 & 0.441 & 0.014 \\
Calpain-1 with CaM & 0.443 & 0.001 & 0.428 \\
Calpain-2 with CaM & 0.402 & 0.003 & 0.401 \\
\hline
\end{tabular}

CaM, calmodulin.

Table III. Cut-off points, sensitivity, specificity and AUC of ROC.

\begin{tabular}{lccccc}
\hline Protein & Cut-off point & Sensitivity, $\%$ & Specificity, $\%$ & LR & AUC of ROC, $\%$ \\
\hline CAPN1 & 0.056 & 25.49 & 94.12 & 4.33 & 56.56 \\
CAPN2 & 0.077 & 52.94 & 92.16 & 6.75 & 75.24 \\
Calpastatin & 0.022 & 86.27 & 66.67 & 2.59 & 76.78 \\
CaM & 0.007 & 72.55 & 86.27 & 5.29 & 83.22 \\
CAPN1 x CAPN2/(CaM x calpastatin) & 25.000 & 84.31 & 86.27 & 6.14 & 89.73 \\
\hline
\end{tabular}

AUC, area under the curve; CAPN1, calpain-1; CAPN2, calpain-2; CaM, calmodulin; ROC, receiver operator characteristic; LR, likelihood ratio.

Table IV. Association between calapin-1, calpain-2, calpastatin and CaM protein expression and clinicopathological variables.

\begin{tabular}{|c|c|c|c|c|c|c|c|c|}
\hline \multirow[b]{2}{*}{ Parameter } & \multicolumn{2}{|c|}{ Calpain-1 } & \multicolumn{2}{|c|}{ Calpain-2 } & \multicolumn{2}{|c|}{ Calpastatin } & \multicolumn{2}{|c|}{$\mathrm{CaM}$} \\
\hline & Normal & Cancer & Normal & Cancer & Normal & Cancer & Normal & Cancer \\
\hline Gender & 0.333 & 0.439 & 0.780 & 0.348 & 0.159 & 0.678 & 0.051 & 0.719 \\
\hline Age & 0.861 & 0.466 & 0.534 & 0.161 & 0.111 & 0.417 & 0.938 & 0.447 \\
\hline Size & 0.376 & 0.946 & 0.479 & 0.519 & 0.330 & 0.619 & 0.122 & 0.134 \\
\hline $\mathrm{T}$ & 0.253 & 0.767 & 0.115 & 0.413 & 0.209 & 0.869 & 0.135 & 0.962 \\
\hline $\mathrm{N}$ & 0.873 & 0.391 & 0.333 & 0.332 & 0.752 & 0.185 & 0.711 & 0.124 \\
\hline cTNM & 0.285 & 0.926 & 0.051 & 0.418 & 0.215 & 0.454 & 0.627 & 0.590 \\
\hline
\end{tabular}

P-values were resultant from unpaired t test (gender) and Spearman's rank correlation (other parameters). No significant P-values were found. CaM, calmodulin; cTNM, clinicopathological stage; T, tumor; $\mathrm{N}$, node.

their correlation with a number of clinicopathological variables, including age, gender, tumor size, postoperative pathological stage and clinicopathological stage (cTNM) (Table IV). None of the proteins investigated in GC or normal tissues showed significant correlation with the clinicopathological variables.

\section{Discussion}

The current study investigated the expression of four calpain system proteins, CAPN1, CAPN2, calpastatin and CaM between GC vs. uninvolved mucosa tissues. The expression level of CAPN2 was higher in GC tissues, while the levels of calpastatin and $\mathrm{CaM}$, which inhibit calpain activity, were higher in uninvolved mucosa tissues. However, CAPN1 protein levels were similar in the two tissues. The alteration of these protein expression levels is in accordance with previous enzyme activity investigation 20). In addition, the alteration of calpain system protein levels was also observed in other types of cancer. In colorectal adenocarcinomas, similar to our results in GC, upregulation of CAPN2 and 
downregulation of calpastatin was observed (17). In human prostate cancer, the mRNA level of CAPN1 increased (22). These results suggested that calpains may be an important factor in tumorigenesis and tumor progression, and higher calpain enzyme activity may be a negative prognostic marker. Studies demonstrated that calpains were responsible for the generation of active forms of proteins that facilitate the progress of cancer: In breast cancer, calpain was associated with the cleavage of human epidermal growth factor receptor 2 (23) and E-cadherin (24), and the two products were responsible for the progression of cancer. In prostate cancer, calpain was hypothesized to be responsible for the generation of active androgen receptor cleavage form (25). In accordance, high-level calpain indicates poor clinical outcome in human breast cancer $(18,26)$. However, in GC Storr et al reported that high level of CAPN1, CAPN2 and calpastatin each indicated a favorable clinical outcome (21). According to the present results, the expression of calpastatin was in positive correlation with calpain in GC. The correlation between the protein level of calpain and its endogenous inhibitor, calpastatin, may be reason that the clapains and its inhibitor, which exert opposite functions, both indicated a favorable prognostic result of GC. Since calpain activity in the prognostic investigation is unknown, whether the higher calpain activity performs a favorable role in $\mathrm{GC}$ requires additional investigation.

To explore whether calpains and their associated proteins may be used as biomarkers that possess diagnostic and treatment potentials, ROC curves were employed in the present study. According to the AUCs (Table III), when using a single protein, $\mathrm{CaM}$ provides the best prediction (AUC $=83.2 \%$ ). In addition, the four proteins in combination, (CAPN1 x CAPN2)/(calpastatin x CaM), improved the prediction ability (AUC $=89.7 \%$ ). Since the ratio of (CAPN1 x CAPN2)/(calpastatin x CaM) may better reflect the calpain activity, the present results suggest that intra-tumor calpain activity may provide a potential biomarker for GC. It has been reported that calpain was responsible for platelet secretion (27). Galectin-3 secretion is also dependent on the calpain activity (28). In addition, calpain itself can be secreted by tubular epithelial cells (29). Thus, detecting serum secreted calpain or screening serum secreting proteins direct or indirect regulated by calpain may provide a new diagnostic target.

The protein expression levels of intra-tumor or normal-appearing gastric mucosal calpain system showed no correlation with various clinicopathological variables, including gender, age, tumor size, $\mathrm{T}$ category, $\mathrm{N}$ category or cTNM. These results are different from Storr's study (21). Whether this system is correlated with the clinicopathological variables requires additional investigation.

In conclusion, calpain may be a positive factor in tumorigenesis and tumor progression. Although the protein expression of all these four proteins was not in association with the clinical variables of GC in the present study, higher calpain enzyme activity may be a negative prognostic marker, since calpains are responsible for the generation of active forms of certain proteins that facilitate the progression of cancer. The ratio of (CAPN1 x CAPN2)/(calpastatin x CaM) may serve as a potential biomarker for GC.

\section{Acknowledgements}

The present study was supported by the National Natural Foundation of China (grant no. 81102460), the College Natural Foundation of Jiangsu Education Department (grant no. 14KJB310026), the Yangzhou Natural Foundation (grant no. YZ2014020), the Fundamental Research Funds for the Central Universities and the Yangzhou University Fundamental Research Funds.

\section{References}

1. Torre LA, Bray F, Siegel RL, Ferlay J, Lortet-Tieulent J and Jemal A: Global cancer statistics, 2012. CA Cancer J Clin 65: 87-108, 2015

2. Chen W, Zheng R, Zeng H, Zhang S and He J: Annual report on status of cancer in China, 2011. Chin J Cancer Res 27: 2-12, 2015.

3. Moretti D, Del Bello B, Allavena G and Maellaro E: Calpains and cancer: Friends or enemies? Arch Biochem Biophys 564: 26-36, 2014.

4. Carragher NO and Frame MC: Calpain: A role in cell transformation and migration. Int J Biochem Cell Biol 34: 1539-1543, 2002.

5. Sorimachi $\mathrm{H}$, Hata $\mathrm{S}$ and Ono Y: Impact of genetic insights into calpain biology. J Biochem 150: 23-37, 2011.

6. Huang Y and Wang KK: The calpain family and human disease. Trends Mol Med 7: 355-362, 2001.

7. Storr SJ, Carragher NO, Frame MC, Parr T and Martin SG: The calpain system and cancer. Nat Rev Cancer 11: 364-374, 2011.

8. Huang Z, Hoffmann FW, Norton RL, Hashimoto AC and Hoffmann PR: Selenoprotein K is a novel target of m-calpain, and cleavage is regulated by Toll-like receptor-induced calpastatin in macrophages. J Biol Chem 286: 34830-34838, 2011.

9. Li X, Li Y, Shan L, Shen E, Chen R and Peng T: Over-expression of calpastatin inhibits calpain activation and attenuates myocardial dysfunction during endotoxaemia. Cardiovasc Res 83: 72-79, 2009.

10. Marshall CB, Nishikawa T, Osawa M, Stathopulos PB and Ikura M: Calmodulin and STIM proteins: Two major calcium sensors in the cytoplasm and endoplasmic reticulum. Biochem Biophys Res Commun 460: 5-21, 2015.

11. Barnes JA and Gomes AV: PEST sequences in calmodulin-binding proteins. Mol Cell Biochem 149-150: 17-27, 1995.

12. Sivanandam A, Murthy S, Chinnakannu K, Bai VU, Kim SH, Barrack ER, Menon M and Reddy GP: Calmodulin protects androgen receptor from calpain-mediated breakdown in prostate cancer cells. J Cell Physiol 226: 1889-1896, 2011.

13. Molinari M, Anagli J and Carafoli E: PEST sequences do not influence substrate susceptibility to calpain proteolysis. J Biol Chem 270: 2032-2035, 1995.

14. Wang KK, Villalobo A and Roufogalis BD: Calmodulin-binding proteins as calpain substrates. Biochem J 262: 693-706, 1989.

15. Yang H, Murthy S, Sarkar FH, Sheng S, Reddy GP and Dou QP: Calpain-mediated androgen receptor breakdown in apoptotic prostate cancer cells. J Cell Physiol 217: 569-576, 2008.

16. Liu T, Mendes DE and Berkman CE: Prolonged androgen deprivation leads to overexpression of calpain 2: Implications for prostate cancer progression. Int J Oncol 44: 467-472, 2014.

17. Lakshmikuttyamma A, Selvakumar P, Kanthan R, Kanthan SC and Sharma RK: Overexpression of $\mathrm{m}$-calpain in human colorectal adenocarcinomas. Cancer Epidemiol Biomarkers Prev 13: 1604-1609, 2004.

18. Storr SJ, Lee KW, Woolston CM, Safuan S, Green AR, Macmillan RD, Benhasouna A, Parr T, Ellis IO and Martin SG: Calpain system protein expression in basal-like and triple-negative invasive breast cancer. Ann Oncol 23: 2289-2296, 2012.

19. Storr SJ, Safuan S, Woolston CM, Abdel-Fatah T, Deen S, Chan SY and Martin SG: Calpain-2 expression is associated with response to platinum based chemotherapy, progression-free and overall survival in ovarian cancer. J Cell Mol Med 16: 2422-2428, 2012.

20. Ivanova EV, Kondakova IV, Spirina LV, Afanas'ev SG, Avgustinovich AV and Cheremisina OV: Chymotrypsin-like activity of proteasomes and total calpain activity in gastric and colorectal cancer. Bull Exp Biol Med 157: 781-784, 2014. 
21. Storr SJ, Pu X, Davis J, Lobo D, Reece-Smith AM, Parsons SL, Madhusudan S and Martin SG: Expression of the calpain system is associated with poor clinical outcome in gastro-oesophageal adenocarcinomas. J Gastroenterol 48: 1213-1221, 2013.

22. Rios-Doria J, Day KC, Kuefer R, Rashid MG, Chinnaiyan AM, Rubin MA and Day ML: The role of calpain in the proteolytic cleavage of E-cadherin in prostate and mammary epithelial cells. J Biol Chem 278: 1372-1379, 2003.

23. Panis C, Pizzatti L, Corrêa S, Binato R, Lemos GF, Herrera AC, Seixas TF, Cecchini R and Abdelhay E: The positive is inside the negative: HER2-negative tumors can express the HER2 intracellular domain and present a HER2-positive phenotype. Cancer Lett 357: 186-195, 2015 .

24. Ye Y, Tian H, Lange AR, Yearsley K, Robertson FM and Barsky SH: The genesis and unique properties of the lymphovascular tumor embolus are because of calpain-regulated proteolysis of E-cadherin. Oncogene 32: 1702-1713, 2013.

25. Libertini SJ, Tepper CG, Rodriguez V, Asmuth DM, Kung HJ and Mudryj M: Evidence for calpain-mediated androgen receptor cleavage as a mechanism for androgen independence. Cancer Res 67: 9001-9005, 2007.
26. Storr SJ, Woolston CM, Barros FF, Green AR, Shehata M, Chan SY, Ellis IO and Martin SG: Calpain-1 expression is associated with relapse-free survival in breast cancer patients treated with trastuzumab following adjuvant chemotherapy. Int J Cancer 129: 1773-1780, 2011.

27. Croce K, Flaumenhaft R, Rivers M, Furie B, Furie BC, Herman IM and Potter DA: Inhibition of calpain blocks platelet secretion, aggregation, and spreading. J Biol Chem 274: 36321-36327, 1999.

28. Menon S, Kang CM and Beningo KA: Galectin-3 secretion and tyrosine phosphorylation is dependent on the calpain small subunit, Calpain 4. Biochem Biophys Res Commun 410: 91-96, 2011.

29. Peltier J, Bellocq A, Perez J, Doublier S, Dubois YC, Haymann JP, Camussi $\mathrm{G}$ and Baud L: Calpain activation and secretion promote glomerular injury in experimental glomerulonephritis: Evidence from calpastatin-transgenic mice. J Am Soc Nephrol 17: 3415-3423, 2006 